\title{
CMV/EBV/ADV/BKV-specific Cytotoxic T Lymphocytes
}

National Cancer Institute

\section{Source}

National Cancer Institute. CMVIEBVIADV/BKV-specific Cytotoxic T Lymphocytes. NCI

Thesaurus. Code C150381.

A population of cytotoxic T-lymphocytes (CT Ls) specifically reactive to cytomeg alovirus $(C M V)$, Epstein-Barr virus (EBV), adenovirus (ADV) and BK virus (BKV), with potential antiviral and protective activities. T-cells derived from peripheral blood mononuclear cells (PBMCs) are exposed to and activated by dendritic cells (DCs) that are loaded with specific peptides from CMV, EBV, ADV and BKV. Upon infusion of the CMV/EBV/ADV/BKV-specific CT Ls, these lymphocytes target and cause lysis of CMV-, EBV-, ADV- and/or BKV-infected cells and may prevent infection and complications from CMV-, EBV-, ADV- and BKV-driven viral diseases. Opportunistic infections caused by these viruses are often seen in immunosuppressed patients. 